Difficulty of comparing the multiple heterogeneous approaches: Comment to Transcranial Direct Current Stimulation in Epilepsy

Markus Gschwind (1,2); Pieter van Mierlo (3)

(1) Department of Neurology, University Hospital Geneva, Geneva, Switzerland

(2) Functional Brain Mapping Laboratory, Department of Neuroscience, Biotech Campus, University of Geneva, Geneva, Switzerland

(3) Medical Image and Signal Processing Group, Electronics and Information Systems Department, Ghent University, Ghent, Belgium

Author's address:

Unité d'EEG et d'exploration de l'épilepsie \& Laboratoire de cartographie cérébrale Service de Neurologie, Hôpitaux Universitaires de Genève (HUG)

Rue Gabrielle-Perret-Gentil 4, 1211 Genève, Switzerland

Phone: +41-79-5533793; Fax +41-22-3728340

Email: markus.gschwind@gmail.com

Words: 368

Figures: 0

Tables: 2

Keywords: transcranial direct current stimulation; tDCS; electrical charge

\title{
Comment to:
}

San-Juan D, Morales-Quezada L, Orozco Garduno AJ, et al. Transcranial Direct Current Stimulation in Epilepsy. Brain Stimul 2015;8:455-64. 
Dear Editor,

We read with pleasure the paper of San-Juan and coworkers [1] published recently in Brain Stimulation. This review has provided a step forward in the direction of the use of this promising technique also in a therapeutic setting with patients suffering from epilepsy. At present, the technical approaches of transcranial direct current stimulation (tDCS) are still very heterogeneous. Nearly every study uses different patient categories, different stimulation protocols, different electrode sizes, stimulation sites and different stimulation current strength, so that comparison between the different studies is limited. It is therefore highly useful to compare all studies, and to provide standardizable measures in order to judge stimulation effects across them.

San-Juan and coworkers have calculated for every study the applied current density and the total electrical charge during stimulation. However, their calculation of the electrical charge is based on an incorrect formula. In the paragraph Data extraction (p.456), they define electrical charge as " $\mathrm{Q}=1$ / $\mathrm{t}$ ", and in both Table 1 and Table 2, they report values of some hundred nanoCoulombs (nC). For example, for the study of Fregni et al. 2006, they report I = $1 \mathrm{~mA}, \mathrm{Q}$ $=833 \mathrm{nC}$ during a total of 20 min stimulation. So they effectively calculated $0.001 \mathrm{~A} /(20 * 60$ $\mathrm{sec})=8.333 \mathrm{e}-7=833.333 \mathrm{e}-9$ Coulomb. They did the like for every reported value of $\mathrm{Q}$. Just above the formula " $Q=1 / t$ ", they also refer to Brunoni et al., 2011 [2]. But those authors defined correctly $Q=I^{*} t$, which is consistent with the definition in physics of the electrical current as the flow rate of electrical charge per time $(I=Q / t=$ Ampere $=$ Coulomb per second). Calculating electrical charge using this correct formula in the same example of Fregni et al 2006 in Table 1, results in $Q=1 \mathrm{~mA} * 20 \mathrm{~min}=0.001 *(20 * 60 \mathrm{sec})=1.2$ Coulomb.

In summary, all values of $Q$ in Table 1 and Table 2 are unfortunately calculated by an incorrect formula and therefore incorrect in values, and 9 orders of magnitude. We think that in the journal Brain stimulation, which is the first address to look for valid reference values in the context of brain stimulation techniques, these wrong values should be corrected (see Table 1 and Table 2 corrected). 


\section{References}

1. San-Juan D, Morales-Quezada L, Orozco Garduno AJ, et al. Transcranial Direct Current Stimulation in Epilepsy. Brain Stimul 2015;8:455-64.

2. Brunoni AR, Amadera J, Berbel B, et al. A systematic review on reporting and assessment of adverse effects associated with transcranial direct current stimulation. Int J Neuropsychopharmacol 2011;14:1133-45.

\section{Acknowledgement}

MG is supported by the Swiss National Science Foundation, grant number 33CM30_124115/2SPUM. PvM is supported by the European Union's Horizon 2020 research and innovation programme under the Marie Sklodowska-Curie grant agreement No 660230. 
Table 1

Summary the safety and efficacy of animals studies using tDCs in epilepsy models.

\begin{tabular}{|c|c|c|c|c|c|c|c|c|c|c|c|c|}
\hline $\begin{array}{l}\text { Author } \\
\text { (year) }\end{array}$ & $\begin{array}{l}\text { Type and } \\
\text { design of } \\
\text { article }\end{array}$ & Animal & $\begin{array}{l}\text { No. of } \\
\text { total } \\
\text { sample }\end{array}$ & $\begin{array}{l}\text { Age } \\
\text { (months) }\end{array}$ & $\begin{array}{l}\text { Sex } \\
\text { (\% males) }\end{array}$ & $\begin{array}{l}I=\text { current; } \\
\text { dosage }(\mathrm{A}) / \\
J=\text { current } \\
\text { density }\left(\mathrm{A} / \mathrm{m}^{2}\right) \\
Q=\text { electrical } \\
\text { charge }(\mathrm{C})\end{array}$ & Montage & $\begin{array}{l}\text { Model of } \\
\text { epilepsy/ } \\
\text { type of } \\
\text { epilepsy }\end{array}$ & $\begin{array}{l}\text { Type and size } \\
\text { of electrodes }\end{array}$ & $\begin{array}{l}\text { Frequency and } \\
\text { duration of session }\end{array}$ & $\begin{array}{l}\text { Adverse } \\
\text { effects }\end{array}$ & Outcome \\
\hline $\begin{array}{c}\text { Liebetanz et al. } \\
\text { (2006) [29] }\end{array}$ & $\begin{array}{l}\text { Original } \\
\text { Experimental }\end{array}$ & Rats & 65 & 2 & 100 & $\begin{array}{l}I_{\max }=100 \mu \mathrm{A} \\
I_{\min }=200 \mu \mathrm{A} \\
J_{\max }=57.142 \mathrm{~A} / \mathrm{m}^{2} \\
J_{\min }=28.571 \mathrm{~A} / \mathrm{m}^{2} \\
Q_{\max , 15 \mathrm{~min}}=222 \mathrm{AC} \\
Q_{\max , 30 \mathrm{~min}}=111 \mathrm{AC} \\
Q_{\max , 60 \mathrm{~min}}=56 \mathrm{nC} \\
Q_{\min , 15 \mathrm{~min}}=111 \mathrm{nC} \\
Q_{\min , 30 \mathrm{~min}}=56 \mathrm{nC} \\
Q_{\min , 60 \mathrm{~min}}=28 \mathrm{nC}\end{array}$ & $\begin{array}{l}2 \mathrm{~mm} \text { left and } \\
2 \mathrm{~mm} \text { anterior } \\
\text { to the bregma }\end{array}$ & $\begin{array}{l}\text { In vivo ramp } \\
\text { model }\end{array}$ & $\begin{array}{l}3.5 \mathrm{~mm}^{2} \\
\quad\left(a=3.5 \times 10^{-6} \mathrm{~m}^{2}\right)\end{array}$ & $\begin{array}{l}4 \text { sessions ( } 50 \mathrm{~Hz} \text {, } \\
2 \text { ms pulse train) } \\
\text { separated by } \\
\text { one week } \\
\text { 1. Cathodal tDCS } \\
\text { for } 30 \text { and for } \\
60 \text { min, anodal } \\
\text { tDCS for } 60 \text { min, } \\
\text { and again } 60 \text { min } \\
\text { of cathodal tDCS. } \\
\text { 2. Cathodal tDCS } \\
\text { for } 15 \text { and for } \\
30 \text { min, anodal } \\
\text { tDCS for } 30 \text { min, } \\
\text { and again cathodal } \\
\text { tDCS for } 30 \text { min. }\end{array}$ & None & $\begin{array}{l}\text { After tDCS, the } \\
\text { threshold } \\
\text { for localized } \\
\text { seizure activity } \\
\text { was determined } \\
\text { repeatedly for } \\
120 \text { min at } \\
\text { intervals of } \\
15 \text { min. } \\
\text { The anticonvulsive } \\
\text { effect induced by } \\
\text { cathodal tDCS } \\
\text { depends on } \\
\text { stimulation } \\
\text { duration and } \\
\text { current strength } \\
\text { and may be } \\
\text { associated with } \\
\text { the induction of } \\
\text { alterations of } \\
\text { cortical excitability } \\
\text { that outlast the } \\
\text { actual stimulation. }\end{array}$ \\
\hline $\begin{array}{l}\text { Kamida et al. } \\
\text { (2011) [31] }\end{array}$ & $\begin{array}{l}\text { Original } \\
\text { Experimental }\end{array}$ & Rats & 18 & 0.7 & 100 & $\begin{array}{l}I=200 \mu \mathrm{A} \\
J=57.142 \mathrm{~A} / \mathrm{m}^{2} \\
Q=111 \mathrm{nC}\end{array}$ & $\begin{array}{l}1.5 \mathrm{~mm} \text { to the } \\
\text { right and } 2 \mathrm{~mm} \\
\text { anterior to the } \\
\text { bregma }\end{array}$ & $\begin{array}{l}\text { In vivo } \\
\text { pilocarpine- } \\
\text { induced } \\
\text { status } \\
\text { epilepticus }\end{array}$ & $\begin{array}{l}\text { 2.1-mm inner } \\
\text { diameter and } \\
3.5 \mathrm{~mm}^{3} \\
\left(a=3.5 \times 10^{-6} \mathrm{~m}^{2}\right)\end{array}$ & 2 weeks; $30 \mathrm{~min}$ & ? & $\begin{array}{l}\text { Neuroprotective } \\
\text { effects on the } \\
\text { immature rat } \\
\text { hippocampus, } \\
\text { including } \\
\text { reduced } \\
\text { sprouting and } \\
\text { subsequent } \\
\text { improvements } \\
\text { in cognitive } \\
\text { performance. } \\
\text { The convulsions } \\
\text { were reduced } \\
21 \% \text { in the } \\
\text { postnatal day } 55 .\end{array}$ \\
\hline
\end{tabular}




\begin{tabular}{|c|c|}
\hline $\begin{array}{l}\text { Tripolar EEG recording } \\
\text { electrode and inner } \\
\text { diameter of } 2.1 \mathrm{~mm} \\
\text { and a contact area } \\
\text { of } 3.5 \mathrm{~mm}^{2} \\
\left(a=3.5 \times 10^{-6} \mathrm{~m}^{2}\right)\end{array}$ & $\begin{array}{l}\text { I. } 10 \text { rats received } \\
4 \text { series of } 15 \text { min } \\
\text { cathodal and anodal } \\
\text { stimulation of } 100 \mu \mathrm{A} \\
\text { with an interval of } 1 \mathrm{~h} \\
\text { and } 45 \text { min in counter } \\
\text { balanced order. } \\
\text { II. } 8 \text { rats received } 4 \\
\text { sessions of } 15 \text { min of } \\
\text { cathodal stimulation } \\
\text { of } 100 \mu \mathrm{A} \\
\text { III. } 8 \text { rats, similar } \\
\text { protocol to II, except } \\
150 \mu \mathrm{A}\end{array}$ \\
\hline
\end{tabular}

hemisphere

with two wires

as ground and

reference on top

epilepsy

protocol to II, except

None I. Neither anodal nor cathodal stimulation had significan long-lasting aftereffects on the number or on the mean duration

of SWDs in the 1-h 45-min

post-stimulation

intervals.

\section{II and III. The} number of

reduced on the stimulation

day compared

to baseline and

increase (II)

or decrease (III)

in the mean

duration of

SWDs from

baseline in

post-stimulation

There were no

significant

differences for

the number

and mean

duration of

SWDs between

the baseline

day and post-

stimulation day

Bilateral cathodal

tDCS, has

short lasting

antiepileptic

effects on the

numbers of

lasting (1-h

lasting (1-h

dependent

effects on the

mean duration of

the spike and

slow-waves

discharges. 
Table 2

Summary the human studies of the safety and efficacy using tDCS in epileptic patients.

\begin{tabular}{|c|c|c|c|c|c|c|c|c|c|c|c|c|}
\hline Author (year) & $\begin{array}{l}\text { Type and design } \\
\text { of article }\end{array}$ & $\begin{array}{l}\text { No. of } \\
\text { total } \\
\text { sample }\end{array}$ & $\begin{array}{l}\text { Age (year } \\
{[\text { mean } \pm \mathrm{SD}} \\
\text { or range]) }\end{array}$ & $\begin{array}{l}\text { Sex (\% } \\
\text { females) }\end{array}$ & $\begin{array}{l}\mathrm{I}=\text { current; dosage } \\
(\mathrm{A}) / J=\text { current } \\
\text { density }\left(\mathrm{A} / \mathrm{m}^{2}\right) / \\
Q=\text { electrical } \\
\text { charge (C) }\end{array}$ & Montage & $\begin{array}{l}\text { Model of } \\
\text { epilepsy/type } \\
\text { of epilepsy }\end{array}$ & $\begin{array}{l}\text { Type and size } \\
\text { of electrodes }\end{array}$ & $\begin{array}{l}\text { Frequency and } \\
\text { duration of } \\
\text { session }\end{array}$ & $\begin{array}{l}\text { Adverse } \\
\text { effects }\end{array}$ & JADDAD & Outcome \\
\hline $\begin{array}{l}\text { Fregni et al. } \\
\text { (2006) [9] }\end{array}$ & $\begin{array}{l}\text { Experimental } \\
\quad \text { randomized } \\
\text { sham controlled } \\
\text { non blinded }\end{array}$ & 19 & $24.16 \pm 7.9$ & 42 & $\begin{array}{l}\mathrm{I}=1 \mathrm{~mA} \\
J=0.285 \mathrm{~A} / \mathrm{m}^{2} \\
Q=833 \mathrm{AC}\end{array}$ & $\begin{array}{l}\text { Cathodal } \\
\text { stimulation } \\
\text { over the } \\
\text { epileptogenic } \\
\text { focus according } \\
\text { to EEG baseline }\end{array}$ & $\begin{array}{l}\text { Focal refractory } \\
\text { epilepsy due to } \\
\text { cortical dysplasia }\end{array}$ & $\begin{array}{l}\text { Sponge electrode } \\
\quad 35 \mathrm{~cm}^{2} \\
\left(a=3.5 \times 10^{-3} \mathrm{~m}^{2}\right)\end{array}$ & $\begin{array}{l}\text { Single session; } \\
20 \mathrm{~min}\end{array}$ & $\begin{array}{l}\text { Itching } \\
\text { (3 active } \\
\text { and 1 sham } \\
\text { groups) }\end{array}$ & 3 & $\begin{array}{l}\text { A significant } \\
\text { reduction } \\
\text { in the number } \\
\text { of epileptiform } \\
\text { discharges } \\
\text { was found } \\
\text { (mean 64.3\%), } \\
\text { however, not } \\
\text { clinical } \\
\text { reduction } \\
\text { of seizure } \\
\text { was seen } \\
\text { in } 30 \text { days } \\
\text { of follow-up. }\end{array}$ \\
\hline $\begin{array}{l}\text { San Juan et al. } \\
\text { (2011) [10] }\end{array}$ & $\begin{array}{l}\text { Case report, } \\
\text { experimental } \\
\text { non controlled } \\
\text { neither blinded }\end{array}$ & 2 & 23 & 0 & $\begin{array}{l}I_{\min }=1 \mathrm{~mA} \\
I_{\max }=2 \mathrm{~mA} \\
J_{\min }=203.018 \mathrm{~A} / \mathrm{m}^{2} \\
J_{\max }=406.091 \mathrm{~A} / \mathrm{m}^{2} \\
Q_{\min }=69 \mathrm{AC} \\
Q_{\max }=139 \mathrm{AC}\end{array}$ & $\mathrm{C} 3, \mathrm{~F} 2$ & $\begin{array}{l}\text { Rasmussen's } \\
\text { encephalitis }\end{array}$ & $\begin{array}{l}\text { Subdermal needle } \\
12 \mathrm{~mm} \text { in length } \\
\text { and } 0.4 \mathrm{~mm} \text { in } \\
\text { diameter } \\
(a=4.925 \times \\
\left.10^{-6} \mathrm{~m}^{2}\right)^{*} \\
\text { *calculating only }^{\text {surface }} \\
\text { area }\end{array}$ & $\begin{array}{l}60 \text { min in four } \\
\text { sessions } \\
\text { (on days } 0, \\
7,30 \text {, and } 60 \text { ) }\end{array}$ & None & 1 & $\begin{array}{l}\text { One patient } \\
\text { was seizure } \\
\text { free and } \\
\text { other } \\
\text { patient with } \\
50 \% \text { of } \\
\text { seizure } \\
\text { frequency } \\
\text { reduction } \\
\text { within } 6 \text { month } \\
\text { of follow-up. }\end{array}$ \\
\hline $\begin{array}{l}\text { Varga et al. } \\
\text { (2011) [11] }\end{array}$ & $\begin{array}{l}\text { Experimental } \\
\text { double blinded } \\
\text { sham-controlled } \\
\text { crossover }\end{array}$ & 5 & $\begin{array}{l}6-11 \\
8.5 \pm 2.5\end{array}$ & 40 & $\begin{array}{l}I=1 \mathrm{~mA} \\
J=0.4 \mathrm{~A} / \mathrm{m}^{2} \\
Q=833 \mathrm{nC}\end{array}$ & $\begin{array}{l}\text { Determined } \\
\text { by visualizing } \\
\text { a 3D voltage- } \\
\text { map of the } \\
\text { focal } \\
\text { epileptiform } \\
\text { discharge }\end{array}$ & $\begin{array}{l}\text { Continuous spikes } \\
\text { and waves } \\
\text { syndrome } \\
\text { during slow } \\
\text { sleep }\end{array}$ & $\begin{array}{l}\text { Sponge electrode } \\
\qquad \begin{array}{l}25 \mathrm{~cm}^{2} \\
\left(a=2.5 \times 10^{-3} \mathrm{~m}^{2}\right)\end{array}\end{array}$ & $20 \mathrm{~min}$ & None & 2 & $\begin{array}{l}\text { Cathodal tDCS did } \\
\text { not reduce the } \\
\text { spike-index in } \\
\text { any of the patients } \\
\text { after } 2 \text { days of } \\
\text { stimulation } \\
\text { session in the } \\
\text { evening; sham } \\
\text { in the first night } \\
\text { and tDCs in the } \\
\text { second night. }\end{array}$ \\
\hline $\begin{array}{l}\text { Yook et al. } \\
\qquad(2011)[12]\end{array}$ & $\begin{array}{l}\text { Case report } \\
\text { Experimental }\end{array}$ & 1 & 11 & 100 & $\begin{array}{l}I=2 \mathrm{~mA} \\
J=0.8 \mathrm{~A} / \mathrm{m}^{2} \\
Q_{20} \mathrm{~min}=1.667 \mu \mathrm{C} \\
Q_{5} \text { days }=8.333 \mathrm{HC} \\
Q_{2} \text { weeks }=16.667 \mathrm{HC}\end{array}$ & $\begin{array}{l}\text { Midpoint } \\
\text { between } \\
\text { P4 and T4 }\end{array}$ & $\begin{array}{l}\text { Bilateral perisylvian } \\
\text { syndrome }\end{array}$ & $\begin{array}{l}\text { Sponge electrode } \\
\qquad 25 \mathrm{~cm}^{2} \\
\quad\left(a=2.5 \times 10^{-3} \mathrm{~m}^{2}\right)\end{array}$ & $\begin{array}{l}5 \text { days a week, } \\
\text { during } \\
2 \text { weeks. } \\
\text { Repeating } \\
\text { procedure } \\
\text { after } \\
2 \text { months; } \\
20 \text { min }\end{array}$ & None & 0 & $\begin{array}{l}\text { During the first two } \\
\text { months after } \\
\text { treatment; the } \\
\text { patient had only } \\
\text { six seizures, with } \\
\text { an evident } \\
\text { clinical } \\
\text { improvement, } \\
\text { after the second } \\
\text { intervention the } \\
\text { patient had just } \\
\text { one seizure } \\
\text { attack over } \\
\text { two months. }\end{array}$ \\
\hline
\end{tabular}




\begin{tabular}{|c|c|c|c|c|c|c|c|c|c|c|c|c|}
\hline $\begin{array}{l}\text { Faria Paula } \\
\text { et al. (2012) } \\
\text { [33] }\end{array}$ & $\begin{array}{l}\text { Cross-over } \\
\text { controlled } \\
\text { trial }\end{array}$ & 2 & 11 and 7 & 0 & $\begin{array}{l}\mathrm{I}=1 \mathrm{~mA} / J=0.285 \\
\mathrm{~A} / \mathrm{m}^{2} \\
\mathrm{Q}=556 \mathrm{AC}\end{array}$ & $\begin{array}{l}\text { Based in } \\
\quad 10-10 \\
\text { International } \\
\text { system } \\
\text { positions } \\
\text { in a cap } \\
\text { (mostly C5-C6) }\end{array}$ & $\begin{array}{l}\text { Drug-refractory } \\
\text { Continuous } \\
\text { Spike-Wave } \\
\text { Discharges } \\
\text { During Slow } \\
\text { Sleep }\end{array}$ & $\begin{array}{l}\text { Sponge electrode } \\
\qquad \begin{array}{l}35 \mathrm{~cm}^{2} \\
\left(a=3.5 \times 10^{-3} \mathrm{~m}^{2}\right)\end{array}\end{array}$ & $\begin{array}{l}\text { Once weekly, } \\
\text { to three } \\
\text { afternoon } \\
\text { sessions of } \\
30 \text { min each. }\end{array}$ & None & 1 & $\begin{array}{l}\text { Cathodal tDCS is } \\
\text { safe and } \\
\text { well-tolerated } \\
\text { in patients with } \\
\text { refractory } \\
\text { epilepsy. They } \\
\text { found a large } \\
\text { reduction in } \\
\text { inter-ictal } \\
\text { epileptiform } \\
\text { EEG discharges } \\
\text { in C5 (mean } \\
32.1 \% \text { ) during } \\
\text { and after the } \\
\text { tDCS (10 min). }\end{array}$ \\
\hline $\begin{array}{l}\text { Auvichayapat } \\
\text { et al. (2013) } \\
\text { [13] }\end{array}$ & $\begin{array}{l}\text { Experimental } \\
\text { randomized } \\
\text { controlled } \\
\text { with sham } \\
\text { unblinded }\end{array}$ & 36 & $6-15$ & 28 & $\begin{array}{l}I=1 \mathrm{~mA} \\
J=0.285 \mathrm{~A} / \mathrm{m}^{2} \\
Q=833 \mathrm{nC}\end{array}$ & $\begin{array}{l}\text { Based in the } \\
\text { international } \\
10-20 \text { EEG } \\
\text { system } \\
\text { (mostly C3-F3) }\end{array}$ & $\begin{array}{l}\text { Focal refractory } \\
\text { epilepsy with } \\
\text { different } \\
\text { etiologies }\end{array}$ & $\begin{array}{l}\text { Sponge electrode } \\
\qquad 35 \mathrm{~cm}^{2} \\
\left(a=2.5 \times 10^{-3} \mathrm{~m}^{2}\right)\end{array}$ & $\begin{array}{l}\text { Single session; } \\
20 \mathrm{~min}\end{array}$ & $\begin{array}{l}\text { One patient } \\
\quad(2.7 \%) \\
\text { developed } \\
\text { a transient } \\
(<2 \mathrm{~h}) \\
\text { erythematous } \\
\text { rash with } \\
\text { no pruritus } \\
\text { or pain under } \\
\text { the reference } \\
\text { electrode }\end{array}$ & 2 & $\begin{array}{l}\text { Cathodal tDCS can } \\
\text { suppress } \\
\text { epileptiform } \\
\text { discharges in } \\
57.6 \% \text { for } 48 \mathrm{~h} \text {, } \\
\text { but the effect } \\
\text { of a single } \\
\text { session on EEG } \\
\text { abnormalities } \\
\text { was not } \\
\text { sustained for } \\
4 \text { weeks. A } \\
\text { statistical } \\
\text { reduction in the } \\
\text { frequency of } \\
\text { seizures was } \\
\text { found (4.8\%) in } \\
\text { the post-hoc } \\
\text { analysis. }\end{array}$ \\
\hline
\end{tabular}

